\title{
The unfriendly ISM in the radio galaxy 4C 12.50 (PKS 1345+12)
}

\author{
R. Morganti ${ }^{1}$, T. A. Oosterloo ${ }^{1}$, C. N. Tadhunter ${ }^{2}$, R. Vermeulen ${ }^{1}$, \\ Y. M. Pihlström ${ }^{3}$, G. van Moorsel $^{3}$, and K. A. Wills ${ }^{2}$ \\ ${ }^{1}$ Netherlands Foundation for Research in Astronomy, Postbus 2, 7990 AA, Dwingeloo, The Netherlands \\ e-mail: morganti@astron.nl \\ 2 Dep. Physics and Astronomy, University of Sheffield, Sheffield, S7 3RH, UK \\ 3 National Radio Astronomy Observatory, Socorro, NM 87801, USA
}

Received 9 April 2004 / Accepted 25 May 2004

\begin{abstract}
The radio source 4C 12.50 has often been suggested to be a prime candidate for the link between ultraluminous infrared galaxies and young radio galaxies. A VLBI study of the neutral hydrogen in the nuclear regions of this object shows that most of the gas detected close to the systemic velocity is associated with an off-nuclear cloud ( $\sim 50$ to $100 \mathrm{pc}$ from the radio core) with a column density of $\sim 10^{22} T_{\text {spin }} /(100 \mathrm{~K}) \mathrm{cm}^{-2}$ and an H I mass of a few times $10^{5}$ to $10^{6} M_{\odot}$. We consider a number of possibilities to explain the results. In particular, we discuss the possibility that this cloud indicates the presence of a rich and clumpy interstellar medium in the centre, likely left over from the merger that triggered the activity and that this medium influences the growth of the radio source. The location of the cloud - at the edge of the northern radio jet/lobe - suggests that the radio jet might be interacting with a gas cloud. This interaction could be responsible for bending the young radio jet. The velocity profile of the gas is relatively broad $\left(\sim 150 \mathrm{~km} \mathrm{~s}^{-1}\right)$ and we interpret this as kinematical evidence for interaction of the radio plasma with the cloud. We also consider the model where the cloud is part of a broader circumnuclear structure. Only a limited region of this structure would have sufficient background radio brightness and large enough column depth in neutral gas to obtain detectable $\mathrm{HI}$ absorption against the counterjet.

The VLBI study of the neutral hydrogen in 4C 12.50 suggests that H I detected near the systemic velocity (as it is often the case in radio galaxies) may not necessarily be connected with a circumnuclear disk or torus (as is very often assumed) but instead could be a tracer of the large-scale medium that surrounds the active nucleus and that may influence the growth of the young radio source.
\end{abstract}

Key words. galaxies: active - galaxies: individual: 4C 12.50 - ISM: jets and outflow - radio lines: galaxies

\section{Introduction}

The onset of radio activity is often thought to be related to an accretion or merger event in the parent galaxy. As the result of such an event, the galaxy harbouring the radio source is likely to have quite a rich interstellar medium (ISM) at least during the initial phase of the AGN. The study of this ISM has a number of interesting aspects. Apart from being a possible source of fuel for the central activity, this ISM is likely to influence the growth and evolution of the radio source as the jets propagate through the ISM on their way out of the galaxy. Secondly, the possible interaction between the radio plasma and the ISM is likely to produce a feedback mechanism that can put substantial amounts of energy into the ISM. This can further influence the evolution of the galaxy as a whole and may even be a factor that regulates the growth of the central black hole (e.g. Silk \& Rees 1998).

There is a consensus now that radio galaxies classified as "Compact Steep Spectrum" (CSS) or "GigaHertz Peaked
Spectra" (GPS) can be identified as objects in their initial phase of radio activity. These radio sources are defined as small (subgalactic, i.e. $\$ 10 \mathrm{kpc}$ ), with steep or convex radio spectra and are very often double lobed, indeed like a mini-radio source (see O'Dea 1998 for a review). Years of observations and discussions have led to the conclusion that these objects are not small because the radio jets are trapped in the surrounding gaseous material, but because they are young (see e.g., Owsianik et al. 1998). However, reaching this conclusion has not been straightforward as there are indications that in these objects interactions between the radio plasma and the ISM do also occur. The ISM may not be trapping the radio source, but it may influence its evolution.

Evidence for some struggle of the radio jet in emerging from the nuclear regions of CSS/GPS comes, for example, from detailed optical studies of a number of cases. Outflows of ionized gas are now unambiguously detected in the two young radio sources PKS 1549-79 and 4C 12.50 (Tadhunter et al. 2001; Holt et al. 2003). Furthermore, broad optical (forbidden) 
emission lines are typically observed in CSS/GPS sources, indicating the presence of gas with disturbed kinematics as a result of an interaction of the radio plasma with the ISM (Gelderman \& Whittle 1994).

In the scenario considered above of radio galaxies originating by merger/interaction events, these characteristics can be explained quite naturally. These young radio sources would be still surrounded by (at least some) material left over from the merger event that triggered the radio source. The study of this medium is, therefore particularly important if we are to understand how the galaxy evolves.

Unfortunately, optical studies of the ionized gas lack the very high spatial resolution that is required to directly infer the details of how the gas is distributed around the nuclear regions of the sources. As this medium is known to be multi-phased from ionized to neutral to molecular - a (complementary) way to study it is through high resolution (VLBI) radio $(21-\mathrm{cm})$ observations of the neutral hydrogen. In this letter we present the results of such a study of the GPS source 4C 12.50. This is a particularly interesting object as it is a prime candidate for the link between ultraluminous infrared galaxies (ULIGs, Sanders \& Mirabel 1996) and radio galaxies. Indeed, the ISM of this radio galaxy is very rich as it is the most far-IR luminous radio galaxy $\left(L_{\mathrm{IR}}=1.7 \times 10^{12} L_{\odot}\right)$ and has a high molecular gas mass $\left(\sim 10^{10} M_{\odot}\right.$, Evans et al. 1999) and a young stellar population component (Grandi 1977; Tadhunter et al. 2004). The substantial contribution found from this stellar component (estimated to have an age between 0.5 and $1.5 \mathrm{Gyr}$ ) implies a major merger as origin of this system. 4C 12.50 is therefore a good candidate in which to study the effects of a rich ISM. The radio source has a size of $\sim 0.15 \operatorname{arcsec}\left(\sim 325 \mathrm{pc}^{1}\right.$ and has all the characteristics of young radio sources (with age $\ll 10^{7} \mathrm{yr}$ ).

H I absorption was detected in 4C 12.50 by Mirabel (1989) using the Arecibo telescope, showing a relatively deep and narrow component $\left(\tau \sim 0.01\right.$ and $\left.F W H M \sim 200 \mathrm{~km} \mathrm{~s}^{-1}\right)$ located close to the systemic velocity as well as a shallower and broader component $\left(\tau \sim 0.002, F W H M \sim 700 \mathrm{~km} \mathrm{~s}^{-1}\right)$. Recent observations done with the new broad-band system at the WSRT have given further insight the characteristics of the absorption. These observations show that the broad absorption is much wider than measured before by Mirabel (1989), covering now a range in velocities of about $2000 \mathrm{~km} \mathrm{~s}^{-1}$; most of this H I absorption is blueshifted (see Morganti et al. 2003a and the profile shown in Fig. 2).

Holt et al. (2003) have found complex optical emission line profiles at the position of the nucleus with line width up to $2000 \mathrm{~km} \mathrm{~s}^{-1}$ and blueshifted $\sim 2000 \mathrm{~km} \mathrm{~s}^{-1}$ with respect to the halo of the galaxy and the deep, narrow component of the $\mathrm{HI}$ absorption. These findings have been interpreted as material in outflow. They support the idea that $4 \mathrm{C} 12.50$ is a young radio source with nuclear regions enshrouded in a dense cocoon of gas and dust and where the radio jets are expanding through this cocoon, sweeping material out from the nuclear regions.

\footnotetext{
${ }^{1}$ For $H_{0}=71 \mathrm{~km} \mathrm{~s}^{-1} \mathrm{Mpc}^{-1}, \Omega_{\mathrm{M}}=0.27$ and $\Omega_{\mathrm{vac}}=0.73$, resulting in a linear to angular scale ratio of $2.165 \mathrm{kpc} \operatorname{arcsec}^{-1}$. Derived using E. L. Wright's cosmology calculator at http://www. astro.ucla.edu/wright/CosmoCalc.html
}

In light of the considerations mentioned above, 4C 12.50 appears to be a good candidate for further study of the influence of the ISM on the evolution of a young radio source. This was, therefore, the goal of our VLBI study of the HI in this radio source.

\section{VLBI observations}

The observations were done using a global VLBI network. This included the VLBA ${ }^{2}$, one antenna of the VLA and the three EVN $^{3}$ telescopes (Effelsberg, Westerbork, Onsala) that can reach the relatively low frequency of the redshifted $\mathrm{HI}$ in 4C 12.50 (1266 MHz) with their $L$-band receivers. The observations were carried out on 24 February 2001. 4C 12.50 was observed for about $9 \mathrm{~h}$. Two calibrators, 3C 273 and 4C 39.25 , were observed, interleaved with the main target.

When these observations were planned, we were not yet aware of the very large width of the H I absorption that was later detected by the WSRT. Therefore, in the VLBI observations we have used an $8 \mathrm{MHz}$ band centred on the frequency of the deep and narrow absorption (1266 MHz). This setup is, therefore, not ideal for the study of the broad component. Thus, in this letter, we will only concentrate on the results from the study of the narrow component. A total number of 256 channels were used given a velocity resolution of about $8 \mathrm{~km} \mathrm{~s}^{-1}$ before Hanning smoothing. The data were correlated at the JIVE correlator in Dwingeloo. The initial calibration used the automatic pipeline developed at JIVE (Reynolds et al. 2002). The data reduction, including bandpass calibration, was done using the Difmap and Miriad packages. A few cycles of phase-only selfcalibration were done until the structure of the source appeared settled. A last iteration with amplitude self-calibration was then performed.

A line data cube was made using natural weighting after subtracting the continuum emission from the $u v$-data using the channels that did not contain the narrow H I absorption. This approach was chosen because the $8 \mathrm{MHz}$ observing band does not allow to determine the properties of the broader absorption. The noise per channel is $\sim 1.3 \mathrm{mJy}^{\text {beam }}{ }^{-1}$ after Hanning smoothing and the restoring beam size is $24 \times 4$ mas $\left(\mathrm{PA}=-11^{\circ}\right)$. The reason for choosing this low resolution is that it allowed the detection of the faint, extended part of the continuum source that turned out to be critical (see below).

A continuum image (at the same spatial resolution) was made using the line-free channels. The noise is $\sim 1 \mathrm{mJy}^{\text {beam }}{ }^{-1}$ and the resulting image is shown in Fig. 1. The well-known, heavily distorted, structure of this source is clearly evident (see also Stanghellini et al. 1997; Xiang et al. 2002; Lister et al. 2003). The location of the core, as derived by Stanghellini et al. (1997) is indicated. The peak of the continuum is $809 \mathrm{mJy} \mathrm{beam}^{-1}$ and the total flux density is $5.17 \mathrm{Jy}$, comparable to previous measurements of the total flux density for

2 The National Radio Astronomy Observatory is operated by Associated Universities, Inc., under cooperative agreement with the National Science Foundation.

3 The European VLBI Network is a joint facility of European, Chinese, South African and other radio astronomy institutes funded by their national research councils. 


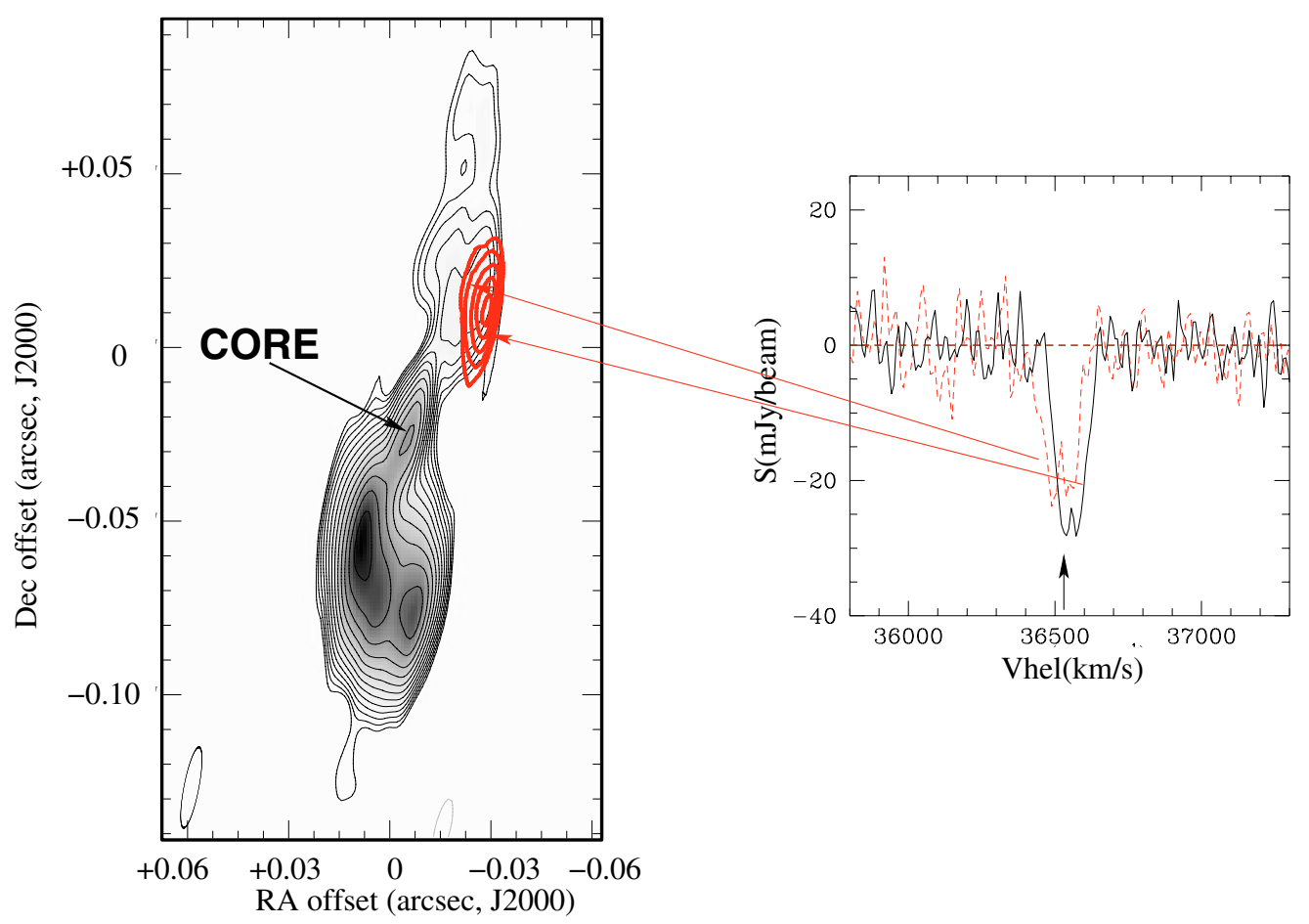

Fig. 1. Continuum image (grey scale and thin contours; negative contour in light grey) of 4C 12.50 with superimposed the total intensity of the H I absorption (thick contours). The absorption profiles at the two sides of the H I cloud are also shown. The position of the radio core as derived by Stanghellini et al. (1997) and confirmed by Lister et al. (2003) is also indicated. The contour levels for the continuum image are: $-5,5 \mathrm{mJy}$ beam $^{-1}$ to $800 \mathrm{mJy}$ beam $^{-1}$ in steps of factor 1.5 . The contour levels for the total intensity of the $\mathrm{H} \mathrm{I}$ absorption are $-2.5,-2.0,-1.5$, $-1.0,-0.5 \mathrm{Jy} \mathrm{beam}^{-1} \mathrm{~km} \mathrm{~s}^{-1}$.

this source. The overall structure is about 150 mas $(\sim 325 \mathrm{pc})$ in size. A possible extension, not seen before, is visible in our continuum image in the northern part of the source (see Fig. 1).

\section{An off-nuclear HI absorption}

The relatively narrow $\mathrm{HI}$ absorption located at the systemic velocity ${ }^{4}$ was clearly detected. Perhaps surprisingly, the absorption is not detected against the core or the brighter southern radio lobe, but instead it is concentrated in a cloud-like structure at the edge of the faint northern lobe. In Fig. 1 the total intensity image of the detected H I absorption is shown superimposed on the continuum emission. The H I cloud extends from about 20 mas $(\sim 43 \mathrm{pc})$ to $\sim 50$ mas $(\sim 108 \mathrm{pc})$ from the nucleus. It appears to be slightly extended with an estimated size of $10 \times 30$ mas (corresponding to $22 \times 65 \mathrm{pc}$ ). A small velocity gradient of $\$ 50 \mathrm{~km} \mathrm{~s}^{-1}$ is observed across the cloud as can be seen from the H I profiles also shown in Fig. 1. The FWHM of the H I profiles (at different positions as shown in Fig. 1) is about $\sim 150 \mathrm{~km} \mathrm{~s}^{-1}$.

Figure 2 shows a comparison between the H I profile derived from the WSRT observations and the VLBI profile integrated over the cloud. From the comparison it appears that, on the VLBI scale, we are detecting basically the entire narrow component of the $\mathrm{HI}$ absorption detected at lower resolution.

\footnotetext{
${ }^{4}$ We use the systemic velocity of $V_{\text {hel }}=36522 \mathrm{~km} \mathrm{~s}^{-1}(z=$ $0.12174 \pm 0.00002)$ derived from the detailed analysis of the emission lines presented in Holt et al. (2003).
}

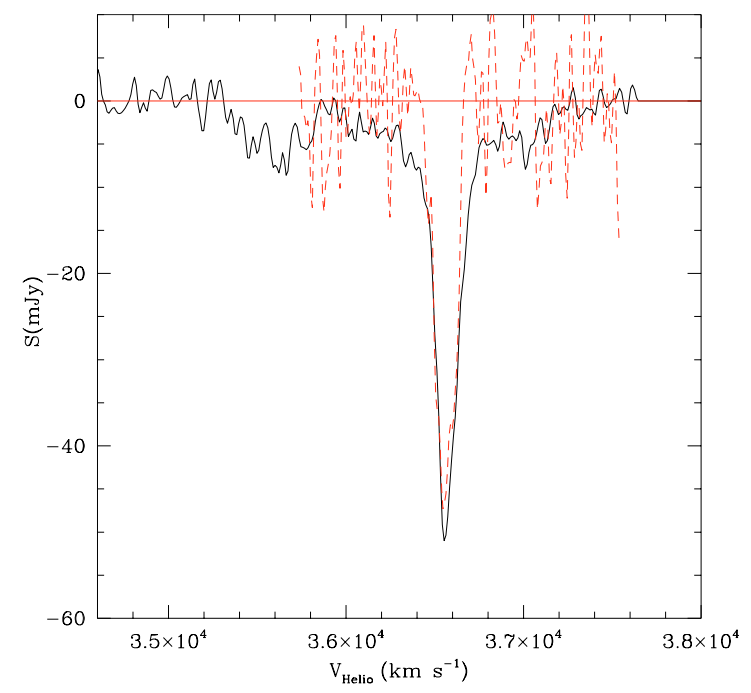

Fig. 2. Comparison between the WSRT profile (black) and the VLBI profile integrated over the H I absorption cloud (dashed).

The HI absorption is located against the edge of the northern radio lobe, against a region of very low brightness radio continuum. The optical depth of the absorption is therefore extremely high. The peak of the HI absorption is $\sim 20 \mathrm{mJy}^{\text {beam }}{ }^{-1}$ and the peak optical depth reaches more than $60 \%$. The corresponding column density of the $\mathrm{HI}$ is up to $\sim 10^{22} T_{\mathrm{spin}} /(100 \mathrm{~K}) \mathrm{cm}^{-2}$. This is likely to be a lower limit to the true column density as the $T_{\text {spin }}$ of a region so close to the AGN can be as high as at least $1000 \mathrm{~K}$ (instead of $100 \mathrm{~K}$ 
which is more typical of the cold, quiescent $\mathrm{H} \mathrm{I}$ in galaxy disks, Maloney et al. 1996). Thus, the true column density will likely be well in excess of $10^{22} \mathrm{~cm}^{-2}$.

\section{Origin of the $\mathrm{HI}$ absorption}

The intriguing result that comes out of these observations is that the $\mathrm{H} \mathrm{I}$ absorption detected at the systemic velocity is originating from a cloud with high optical depth situated (in projection) between 40 and $100 \mathrm{pc}$ away from the core.

$\mathrm{HI}$ detected at the systemic velocity is often considered to be associated with a circumnuclear disk or torus (see e.g., Conway \& Blanco 1995; Peck \& Taylor 2001). Given the observed characteristics on the VLBI scale, this interpretation is not at all obvious in the case of the H I detected in 4C 12.50. This suggests that some care should be taken when interpreting H I absorption data from low resolution observations, before the spatial information provided by the VLBI is available.

The HI absorption is located at the edge of the northern lobe/jet, in a region of relatively weak radio continuum. This suggests that the medium producing the absorption is quite inhomogeneous with low filling factor, since otherwise absorption would have been detected in other locations. The H I column depth continues to increase up to the edge of the radio source. This suggests that there may be more atomic gas further to the south and/or west, where it can no longer be seen in front of any radio emission. Thus, while the visible extent of the absorber is about $22 \times 65 \mathrm{pc}$, its actual size could easily be larger.

We find the properties of this absorber somewhat remarkable, and below we describe several possible geometrical scenarios, none of which is entirely conclusive.

\subsection{A circumnuclear structure}

A first possibility is that the visible absorbing region is indeed not an isolated cloud, but forms part of a broader circumnuclear structure around the centre, as expected from the traditional orientation-unification models (see e.g., Antonucci 1993). This structure occupies a roughly toroidal region around the centre, through which the radio jets protrude into roughly the polar directions. It could be that there is only a limited region, in the northwestern sector, where there is both sufficient background radio brightness and which has a large enough column depth in neutral gas to obtain detectable H I absorption against the counterjet. A modest misalignment between the axes of the jets and the torus is then indicated, but if the torus is geometrically quite thick, we could be seeing only a region near its northern edge, while the atomic gas density could increase towards the southwest, as surmised above, but be invisible there for lack of radio background.

The entire southern radio structure could be protruding in front of the torus in our direction, while at small (projected and physical) distances to the core, the torus may no longer have a large enough column depth of atomic gas (it could be ionised, see e.g the case of NGC 1052, Vermeulen et al. 2003). A plausible projected position angle for the torus could be in the range 90-120 deg. We do not see the misalignment between this structure, on scales of tens to a hundred pc, and the inner jets, which presumably are first collimated by a central engine in an accretion disk geometry on much smaller scales, as a fatal objection to the model. Indeed, whether or not jet engine precession also plays a role (Lister et al. 2003), one could speculate that the fact that the outer jets, on scales of tens to a hundred pc, are more closely perpendicular to the surmised torus, is an indication of some bending they undergo under the influence of the same density and pressure gradient of which the torus is a manifestation.

However, the fact that we recover most of the flux detected at low resolution indicates that the lack of more extended $\mathrm{HI}$ absorption is not due to lack of sensitivity. It is worth mentioning that in the other cases of $\mathrm{HI}$ absorption associated with a circumnuclear structure (e.g. 1946+708, Cygnus A, Hydra A and 4C 31.04 studied by Peck \& Taylor 2001; Conway 1999; Taylor 1996 and Conway 1997 respectively or even Arp 220, Mundell et al. 2001) the source appears to be more homogenously covered by the H I screen instead of showing only one region of very high optical depth.

\subsection{An isolated HI cloud: Interaction or chance alignment?}

We now consider the possibility that the detected $\mathrm{H} \mathrm{I}$ is coming from an isolated cloud. As mentioned above, the H I cloud revealed by the new VLBI observations is intriguingly located at the edge of the northern lobe/jet. This can be, of course, just a projection effect. The transverse, projected distance of the absorber from the centre is of order $50 \mathrm{pc}$ but the cloud could be radially along our line of sight at a much greater distance to the core, potentially even at a kpc or more. However, it must then be part of a distribution of clouds with a low enough number density and volume filling factor that we see no other, potentially even a factor 40 lower column depth clouds at similar distances in front of the larger, brigher southern radio jet/lobe structure. In other words, the projection of this cloud over the edge of the counterjet is fortuitous in this hypothesis. However, it is also worth mentioning that, as noted before in the case of other H I absorption studies (see e.g. Peck \& Taylor 2001), the $F W H M$ of the detected $\mathrm{HI}$ is significantly greater than that seen toward the centre of our Galaxy $\left(\sim 20 \mathrm{~km} \mathrm{~s}^{-1}\right.$, van der Hulst et al. 1983) and so it seems unlikely that it is simply a cloud in the host galaxy that happens to lie on the line of sight of the central radio source.

Alternatively, the observed $\mathrm{H}$ I absorber could have a lineof-sight distance to the core similar to its projected distance, about $50 \mathrm{pc}$. Potentially, the radio counterjet could then be interacting with the atomic gas. In this context, it appears remarkable that the cloud is located in the region where the northern jet appears to slightly bend and become less collimated.

A kinematical analysis has led Lister et al. (2003) to suggest that $4 \mathrm{C} 12.50$ has a conical helical jet. This could arise from orbital motion of the black-hole and/or precession of the jet nozzle. If this is the case, the bending of the jet in the northern region could be due to such a precession more than to some 
interaction with the external medium. Although one may wonder, given that the size of the cloud is comparable to the projected distance to the centre, whether such a cloud could be dynamicaly stable, the location of the H I cloud suggests that it is worth at least considering the possibility of a physical association between the radio jet and the H I cloud suggesting that the cloud could be responsible for bending the jet.

On the other hand, an even sharper bend is observed in the southern jet and one may wonder why no $\mathrm{HI}$ absorption is detected there. However, only gas that is in front of the radio source is of course detected via absorption. The geometry and the location of the clumps is therefore extremely critical.

Regardless whether a direct interaction is actually occuring, the presence of such a cloud suggests that the medium around the central regions of $4 \mathrm{C} 12.50$ must be formed by dense and clumpy structures. The presence of a clumpy medium is also indicated by the polarization study. Isolated regions of high fractional radio polarization - detected only when observed with the high resolution of the VLBI - were found by Lister et al. (2003) suggesting the presence of a clumpy (and depolarizing) medium.

\section{Effects of a clumpy medium}

In Sect. 4 we have shown that, although we cannot rule out completely other possible explanations, a likely possibility is that we have detected an high column density $\mathrm{HI}$ cloud in the central region $(\sim 50 \mathrm{pc})$ of $4 \mathrm{C} 12.50$ against which the jet is interacting.

The presence of a medium formed by dense clumps surrounding the nuclear regions can be expected, at least in the first stage of the radio galaxy's evolution, as a result of a merging event. Clouds like the one detected in the $\mathrm{HI}$ observations of 4C 12.50 could represent the debris left over from the merger that triggered the activity. The presence of a clumpy medium can therefore be a relatively common phenomena (see Sect. 6) that needs to be taken into account in order to understand the evolution of the initial phase of a newly-born (or restarted) radio jet.

In order to understand what would be the effect of this cloud on the jet, we can estimate the mass of the HI in the cloud. As mentioned above, the minimum estimated size is $22 \times 65$ pc. Assuming an average column density of $6 \times 10^{21}$ $\mathrm{cm}^{-2}$ (which is a strict lower limit), we estimate the H I mass to be $\sim 7 \times 10^{4} M_{\odot}$. As discussed above, the column density could be easily a factor 10 higher, so this will give a few times $10^{5}$ to $10^{6} M_{\odot}$ as H I mass. This is not too different from the mass derived for the ionized gas (see Holt et al. 2003). However, even if a direct interaction occurs, the amount of gas does not seem to be enough to frustrate the radio jet (see Carvalho 1994, 1998; one would need more than $10^{10} M_{\odot}$ ). It could, however, be able to momentarily destroy the path of the jet and therefore slow down the growth of the radio source until the radio plasma clears its way out. The similarities between the radio morphology of 4C 12.50 and the simulation of a jet interacting with a clumpy medium (Bicknell et al. 2003) are indirect support of this scenario. In these simulations, the jet is temporarely disrupted and decollimated due to the interaction with a dense cloud in the ISM. After a while this cloud is destroyed and the jet collimates again and can continue in its expansion.

Evidence that this could indeed be the situation is suggested also by the study of the ionized gas (Holt et al. 2003). In 4C 12.50 complex emission line profiles are observed at the position of the (optical arcsec-scale) nucleus. They have been interpreted as resulting from emission originating from kinematically distinct regions with gradients in density, ionisation potential and velocity. This is what is expected if we are dealing with a young radio galaxy with nuclear regions that are still enshrouded in a dense cocoon of gas and dust and with the radio jets now expanding through this cocoon, sweeping material out of the nuclear regions. More examples of radio galaxies in such a stage of their evolution do exist (see e.g. PKS 1549-79, Tadhunter et al. 2001).

As mentioned in the introduction, the presence of a rich ISM in 4C 12.50 is well known, e.g., from the high concentration of molecular gas found by Evans et al. (1999) as well as the high far-IR luminosity and a young stellar population component. Because of this, $4 \mathrm{C} 12.50$ has been often been suggested as a prime candidate for the link between majormerger systems like the ultraluminous infrared galaxies and radio galaxies.

In the case of nearby large mergers like the Antennae, molecular gas has been found in the form of giant molecular clouds. Following the work of e.g. Wilson et al. (2003), those clouds can be relatively large (up to a few hundred pc in size), with a $\triangle F W H M \sim 20-50 \mathrm{~km} \mathrm{~s}^{-1}$ and a mass range between $5 \times 10^{6}$ and $9 \times 10^{8} M_{\odot}$. The H I cloud we detect is smaller than this and less massive. However, if the H I corresponds only to that part of the gas cloud that is photo-dissociated by the radiation from the AGN or the shocks produced by the interaction with the radio jet, the total gas mass may fall in the same range. A major difference is the width of the line: the H I cloud in $4 \mathrm{C} 12.50$ shows a much wider profile. This could be an indication of a kinematic disturbance due to the jet and further evidence that this gas is indeed affected by some interaction with the radio jet.

More gas clouds could be in the path of the radio plasma flow and, in particular, some of them can be already "run over" by the jet. If that happens, the clouds are compressed and they fragment. The fragments can quickly radiate away their excess energy. This results in the formation of dense and cool structures at high velocities (Mellema et al. 2002). This part of the gas can be responsible for the broad $\mathrm{HI}$ absorption (detected in the WSRT data), and be similar to the case of 3C 293 described in Morganti et al. (2003c).

\section{A common phenomenon?}

4C 12.50 does not represent the first case where the presence of H I absorption is associated with an off-nuclear cloud. One of the best examples known is the Seyfert galaxy IC 5063 (Morganti et al. 1998, 2003b; Oosterloo et al. 2000) where the off-nuclear $\mathrm{HI}$ absorption is associated with a region of interaction between the radio jet and the ISM (in particular a molecular cloud). In IC 5063, the presence of interaction is further 
supported by the fact that the absorption is highly blueshifted (up to $700 \mathrm{~km} \mathrm{~s}^{-1}$ ), and therefore corresponds to a fast outflow.

Among radio galaxies, 3C 236 shares many similarities with 4C 12.50. Although it is a giant radio galaxy, it shows a restarted activity as indicated by the nuclear component that has a structure similar to many CSS sources (Schilizzi et al. 2001). The VLBI study carried out by Conway \& Schilizzi (2000) has shown that relatively narrow, but high opacity, absorption is associated with the tip of the eastern jet (about $2 \mathrm{kpc}$ away from the nucleus). Conway \& Schilizzi (2000) explain this absorption as due to the photo-dissociation of molecular gas by the radiation from the shock produced by interaction between the radio jet and a molecular cloud. Further down along the lobe, a broader component of the H I absorption is seen, indicating (according to Conway \& Schilizzi 2000) that those regions have been reached and disturbed by the shock.

Another, more recent, example of off-nuclear HI absorption has been found in the Compact Symmetric object 4C 37.11 (or 0402+379, see Maness et al. 2004) where broad $\left(\sim 500 \mathrm{~km} \mathrm{~s}^{-1}\right.$, but in this case redshifted compared to the systemic) absorption line was found in the region of the southern hot-spot.

\section{Conclusions}

VLBI observations of neutral hydrogen in the radio galaxy 4C 12.50 have given that perhaps surprising result that most of the HI detected close to the systemic velocity is concentrated in a high column density cloud situated - in projection at about $50 \mathrm{pc}$ from the nucleus.

The radio activity in $4 \mathrm{C} 12.50$ is considered to be triggered by the merger of gas-rich galaxies for a number of reasons (e.g. the large amount of molecular gas detected, by Evans et al. 1999). This is also confirmed by the study of the stellar population of the host galaxy (Tadhunter et al. 2004), where a substantial contribution from a young stellar component is found, implying a major merger as origin of this system. The young stellar component is, however, relatively old (between 0.5 and $1.5 \mathrm{Gyr}$ ) suggesting that the activity was triggered late in the merger process.

The H I cloud detected in 4C 12.50 indicates, indeed, that, long after the merger took place, a clumpy interstellar medium is present in the nuclear regions of the galaxy. The presence of such a clumpy medium supports the idea (also suggested by the observations of ionized gas) that the radio jets may experience strong interaction with this medium before the radio plasma clears its way out. Clouds like the one detected in $4 \mathrm{C} 12.50$ are likely to be responsable for the bending and decollimation of the young radio jet. A similar distribution of the ISM may be common in radio galaxies for which the radio activity just started (or restarted, as it is the case in 3C 236).

Finally, the VLBI observations of the neutral hydrogen in 4C 12.50 have shown that $\mathrm{HI}$ absorption detected close to the systemic velocity in many radio galaxies cannot be taken (by itself) as evidence for being associated with circumnuclear tori or disks, but high resolution follow up are always necessary to get better understanding of the actual origin of this gas.

\section{References}

Antonucci, R. 1993, ARA\&A, 31, 473

Bicknell, G., Saxton, C. J., Sutherland, R. S., Midgley, S., \& Wagner, S. J. 2003, New Astron. Rev., 47, 537

Carvalho, J. C. 1994, A\&A, 292, 392

Carvalho, J. C. 1998, A\&A, 329, 845

Conway, J. E., \& Blanco, P. R. 1995, ApJ, 449, L131

Conway, J. E. 1997, in 2nd Workshop on GPS and CSS radio sources, ed. I. A. G. Snellen et al. (Leiden: Publ JIVE), 198

Conway, J. E. 1999, in Highly redshifted radio lines, ed. C. L. Carilli et al., ASP Conf. Ser., 156, 259

Conway, J. E., \& Schilizzi, R. T. 2000, Proc. of the 5th european VLBI Network Symp., ed. J. E. Conway, A. G. Polatidis, R. S. Booth, \& Y. M. Pihlström, Onsala Space Observatory, 123

Evans, A., Kim, J. M., Mazzarella, J. M., Scoville, N. Z., \& Sanders, D. B. 1999, ApJ, 521, L107

Gelderman, R., \& Whittle, M. 1994, ApJS, 91, 491

Grandi, S. A. 1977, ApJ, 215, 446

Holt, J., Tadhunter, C., \& Morganti, R. 2003, MNRAS, 342, 227

Lister, M. L., Kellermann, K. I., Vermeulen, R. C., et al. 2003, ApJ, 584,135

Maness, H. L., Taylor, G. B., Zavala, R. T., Peck, A. B., \& Pollack, L. K. 2004, ApJ, 602, 123

Maloney, P. R., Hollenbach, D. J., \& Tielens, A. G. G. M. 1996, ApJ, 466, 561

Mellema, G., Kurk, J. D., \& Röttgering, H. J. A. 2002, A\&A, 395, L13

Mirabel, I. F. 1989, ApJ, 340, L13

Morganti, R., Oosterloo, T., Emonts, B. H. C., Tadhunter, C. N., \& Holt J. 2003a, Recycling Intergalactic and Interstellar Matter, ed. P.-A. Duc, J. Braine, \& E. Brinks, in press Proc. IAU Symp., 217 [arXiv:astro-ph/0310629]

Morganti, R., Oosterloo, T., Holt, J., Tadhunter, C., \& van der Hulst, J. M. 2003b, ESO Messenger, 113, 69 (http://www . eso.org/gen-fac/pubs/messenger/)

Morganti, R., Oosterloo, T. A., Emonts, B. H. C., van der Hulst, J. M., \& Tadhunter, C. 2003c, ApJ, 593, L69

Morganti, R., Oosterloo, T., \& Tsvetanov, Z. 1998, AJ, 115, 915

Mundell, C. G., Ferruit, P., \& Pedlar, A. 2001, ApJ, 560, 168

O’Dea, C. P. 1998, PASP, 110, 493

Oosterloo, T. A., Morganti, R., Tzioumis, A., et al. 2000, AJ, 119, 2085

Owsianik, I., Conway, J. E., \& Polatidis, A. 1998, A\&A, 336, L37

Peck, A. B., \& Taylor, G. B. 2001, ApJ, 554, L147

Reynolds, C., Paragi, Z., \& Garrett, M. A. 2002, Pipeline processing of VLBI data in Proc. of the XXVIIth General Assembly of the International Union of Radio Science (Gent: URSI), session J8.P.4, paper No. 0924 [arXiv: astro-ph/0205118]

Silk, J., \& Rees, M. J. 1998, A\&A, 331, L1

Schilizzi, R. T., Tian, W. W., Conway, J. E., et al. 2001, A\&A, 368, 398

Stanghellini, C., O’Dea, C. P., Baum, S. A., et al. 1997, A\&A, 325, 943

Tadhunter, C. N., Wills, K. A., Morganti, R., Oosterloo, T., \& Dickson, R. 2001, MNRAS, 327, 227

Tadhunter, et al. 2004, MNRAS, submitted

Taylor, G. B. 1996, ApJ, 470, 394

Vermeulen, R. C., Ros, E., Kellermann, K. I., et al. 2003, A\&A, 401, 113

Xiang, L., Stanghellini, C., Dallacasa, D., \& Haiyan, Z. 2002, A\&A, 385,768

Wilson, C. D., Scoville, N., Madden, S. C., \& Charmandaris, V. 2003, ApJ, 599, 1049 